\title{
Cis-interactions between Notch and its ligands block ligand-independent Notch activity
}

\author{
William Hunt Palmer, Dongyu Jia, Wu-Min Deng* \\ Department of Biological Science, Florida State University, Tallahassee, United States
}

\begin{abstract}
The Notch pathway is integrated into numerous developmental processes and therefore is fine-tuned on many levels, including receptor production, endocytosis, and degradation. Notch is further characterized by a twofold relationship with its Delta-Serrate (DSL) ligands, as ligands from opposing cells (trans-ligands) activate Notch, whereas ligands expressed in the same cell (cis-ligands) inhibit signaling. We show that cells without both cis- and trans-ligands can mediate Notch-dependent developmental events during Drosophila oogenesis, indicating ligand-independent Notch activity occurs when the receptor is free of cis- and trans-ligands. Furthermore, cis-ligands can reduce Notch activity in endogenous and genetically induced situations of elevated trans-ligand-independent Notch signaling. We conclude that cis-expressed ligands exert their repressive effect on Notch signaling in cases of trans-ligand-independent activation, and propose a new function of cis-inhibition which buffers cells against accidental Notch activity.
\end{abstract}

DOI: 10.7554/eLife.04415.001

*For correspondence: wumin@ bio.fsu.edu

Competing interests: The authors declare that no competing interests exist.

Funding: See page 10

Received: 19 August 2014 Accepted: 06 December 2014 Published: 08 December 2014

Reviewing editor: Utpal Banerjee, University of California, Los Angeles, United States

(c) Copyright Palmer et al. This article is distributed under the terms of the Creative Commons Attribution License, which permits unrestricted use and redistribution provided that the original author and source are credited.

\section{Main text}

Canonical Notch signaling begins when the Notch receptor receives a stimulus from a DSL-type ligand (Delta [DI] or Serrate [Ser] in Drosophila) in an adjacent cell, which leads to $y$-secretase-dependent cleavage of Notch, and translocation of the intracellular domain- $\mathrm{N}^{\mathrm{ICD}}$ - into the nucleus to act as a transcriptional co-activator (de Celis, 2013). Notch may also be activated in a non-canonical, DSLligand independent manner (Hori et al., 2012). DSL ligands can cis-inhibit ligand-dependent Notch activation when expressed in the same cell as the receptor (Micchelli et al., 1997; Del Álamo et al., 2011). However, the possibility of a relationship between DSL-ligand independent Notch activation and cis-expressed ligands has not been explored.

The developing Drosophila egg chamber is a convenient model for dissecting the effects of Notch ligands in cis and in trans, as Dl is the sole signaling source and the signal sending and receiving cells can be easily distinguished (Deng et al., 2001; López-Schier and St Johnston, 2001). (Figure 1-figure supplement 1 provides a brief schematic depiction of the stages of early oogenesis.) At oogenesis stage 7, Notch signaling is activated in the somatic follicle cells by a robust germline Dl upregulation, which leads to the expression of Hindsight (Hnt), downregulation of Cut, and the polyploidization of the follicle cells (Deng et al., 2001; López-Schier and St Johnston, 2001; Sun and Deng, 2005, 2007) (Figure 1A). When Dl germline mutant clones were generated (i.e., trans-activation was removed), the follicle cells failed to downregulate Cut expression, which persisted past stage 7, indicative of a failure to activate Notch (Figure 1B). In contrast, DI follicle cell mutant clones show precocious Cut downregulation at stage 6 attributable to the relief of cis-inhibition (Poulton et alo, 2011) (Figure 1C). Surprisingly, DI mutant clones in the follicle cells bordering DI mutant clones in the germline (i.e., a germline with no signaling source, herein referred to as DI-/DI-cells) show correct Hnt and Cut expression from stage 7 (Figure 1D,E, Figure 1-figure supplement 2A,B). These DI-/DIclones also correctly transit into the endocycle, as their nuclear volumes are similar to wild-type follicle cells in the later stages of oogenesis after polyploidization (Figure 1F, G), whereas cells neighboring 
eLife digest Many biological processes require cells to send messages to one another. Typically, this is achieved when molecules are released from one cell and make contact with companion molecules on another cell. This triggers a chemical or biological reaction in the receiving cell.

One of the most common examples of this is the Notch pathway, which is used throughout the animal kingdom and plays an important role in helping cells and embryos to develop. The Notch protein itself is a 'receptor' protein that is embedded in the surface of a cell, and relays signals from outside the cell to activate certain genes inside the cell. In fruit flies, two proteins called Serrate and Delta act as 'ligands' for Notch-by binding to Notch, they can change how this receptor works.

If Serrate or Delta are present on the outside of one cell, they can activate Notch (and hence the Notch signaling pathway) in an adjacent cell. However, if the Serrate or Delta ligands are present on the surface of the same cell as Notch they turn the receptor off, rather than activate it. Notch can also work without being activated by Serrate or Delta, but whether the ligands can inhibit this 'ligandindependent' Notch activation if they are on the surface of the same cell as the Notch receptor was unknown.

Palmer et al. study Notch signaling in the fruit fly equivalent of the ovary, in cells that are naturally deficient in Serrate and from which Delta was artificially removed. The Notch protein was activated when these ligands were not present. Furthermore, the developmental processes that are activated by Notch were able to proceed as normal when triggered by ligand-independent Notch signaling. In total, Palmer et al. investigated three different types of fruit fly cell, and found that ligandindependent Notch signaling can occur in all of them.

Reintroducing Delta to the same cell as Notch turns the receptor off, suggesting that ligands on the surface of the same cell as the receptor can inhibit ligand-independent Notch activity. Many genetic diseases and cancers have been linked to Notch being activated when it should not be; therefore, understanding how Notch is controlled could help guide the development of new treatments for these conditions. DOI: 10.7554/eLife.04415.002

DI-/Dl-follicle cell clones (retaining a cis-ligand but without a trans-ligand) are comparable to wild-type cells before entry to endocycle (Figure 1F,G). Removal of both cis- and trans-Dl through knockdown of $\mathrm{DI}$ by RNA interference (RNAi) simultaneously in the germline and soma confirmed this finding (Figure 2-figure supplement 1A,B). Together, these observations provide evidence that follicle cells without both cis- and trans-ligand sources can still enter the endocycle stages of oogenesis. This back-up route to the endocycle is not a co-option of Ser in place of DI, as D/RevF10 $\operatorname{Ser}^{R \times 82}$ double clones recapitulated the DI-/DI- phenotype (Figure 1E, Figure 1-figure supplement 2A).

To determine whether the entry into the endocycle in $D I-/ D I$ - follicle cells still requires the function of Notch, we implemented the mosaic analysis with a repressible cell marker (MARCM) system (Lee and Luo, 2001). The MARCM system enables us to create mutant clones while driving expression of a UAS transgene specifically in those clonal cells. DI-/DI- clones driving expression of Notch ${ }^{R N A i}$ show a significantly higher proportion ( $p<0.0001$ ) of late Cut-expressing cells than the DI-/DI- clones alone, indicating that Notch is still required for the mitotic-to-endocycle switch (Figures 1D and 2A, Figure 1-figure supplement 2C, Figure 2-figure supplement 1C, Supplementary file 1). Likewise, MARCM clones for the null allele of Suppressor of Hairless (Drosophila Notch transcriptional effector), $\mathrm{Su}(\mathrm{H})^{47}$, in RNAiinduced DI-/DI- clones also show late Cut expression $(p<0.0001)$ (Figure 2B, Figure 1-figure supplement 2C, Supplementary file 1) in comparison with RNAi-induced DI/DI- clone controls (Figure 2-figure supplement 1A,D). A Notch activity reporter, Notch Responsive Element (NRE)green fluorescent protein (GFP) (Stempfle et al., 2010) was also upregulated in DI-/Dl-clones as early as stage 2, and this expression persisted beyond stage 6 (Figure 2C,D), suggesting that NRE-GFP is probably more sensitive to Notch activation than $\mathrm{Hnt}$ in follicle cells. Together, these results suggest that Notch activity occurs independently of canonical ligands when both cis- and trans-ligands are removed, resulting in normal downstream developmental events in the follicle cells. Consistently, $\left.D\right|^{R e v F 10} S e r^{R \times 82}$ double mutant clones in the wing and eye discs show a slight cell-autonomous upregulation of NRE-GFP in the clone center, which would only occur if cis-inhibition blocked a DSL-independent mode of Notch activity, as interior cells have no access to trans-ligand (Figure 2E,F). This NRE-GFP 

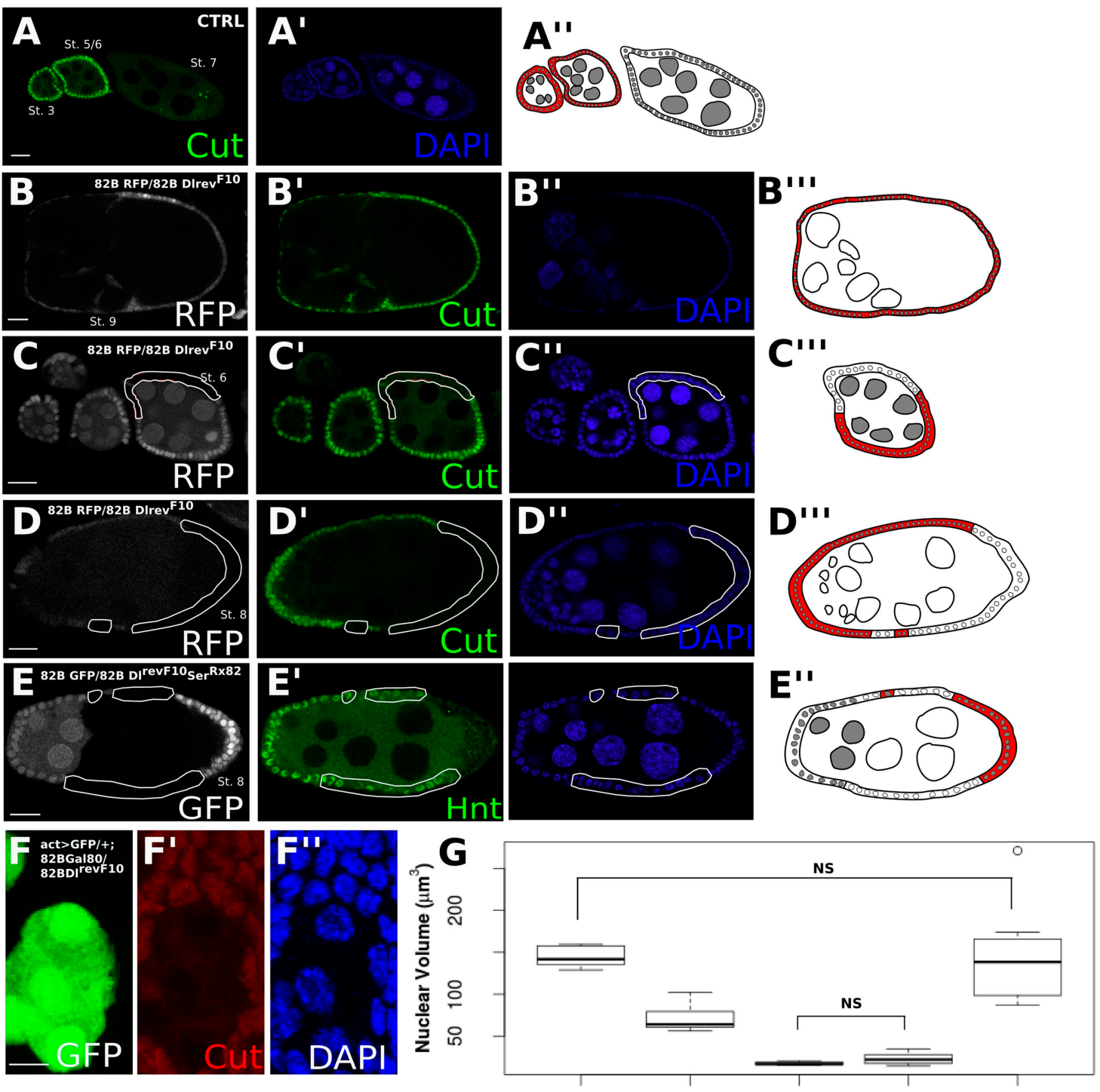

WT St. 10B WT St. 9

WT St. 6 DI GL Clone

DI-/DI- Clone

Figure 1. Follicle cells without DSL ligand bordering germline cells without DSL ligand show proper Notch activation and downstream differentiation. Illustrations legend: active Notch = white cytoplasm, inactive Notch = red cytoplasm, WT cell = grey nuclei, mutant clone = white nuclei. (A-E). Follicle cells downregulate Cut at stage 7 of oogenesis (A). DrrevF10 mutant germline cells cause late Cut expression in follicle cells (B). DlrevF10 mutant follicle cells downregulate Cut early (C). D revF10 follicle cell clones bordering DlrevF10 germline clones show proper Cut downregulation (D). DlrevF10Ser ${ }^{R \times 82}$ mutant follicle cell clones bordering DlrevF10 Ser ${ }^{R \times 82}$ germline clones also show proper Hnt (E). See Figure 1-figure supplement 2 for a z-series image for 1D and 1E. These germline/follicle cell clones ( $\mathbf{D}$ and $\mathbf{E}$ ) show increased nuclear size comparable to wild-type (WT) follicle cells which have entered the endocycle $(n=8$ for each stage/genotype) ( $\mathbf{F}$ and $\mathbf{G})$. For $(\mathbf{G})$, Welch t-tests were done to assess significance between each condition. The only comparisons that were not significant were between WT stage $10 \mathrm{~B}$ and DI-/DI- clones and between WT stage 6 and D/ germline clones, indicating nuclear size in germline clones alone is similar to that of cells before the endocycle, whereas DI-/DI- clonal nuclei are more similar in size to cells that have entered the endocycle. Scale bars represent $20 \mu \mathrm{m}$, except in $\mathbf{F}$, where the scale bar represents $5 \mu \mathrm{m}$.

DOI: 10.7554/eLife.04415.003

The following figure supplements are available for figure 1:

Figure supplement 1. A schematic depiction of the early stages of Drosophila oogenesis.

DOI: 10.7554/eLife.04415.004

Figure supplement 2. Z-stacked images of DI-/DI- clones and quantification of Cut staining in egg chamber clones.

DOI: 10.7554/eLife.04415.005 

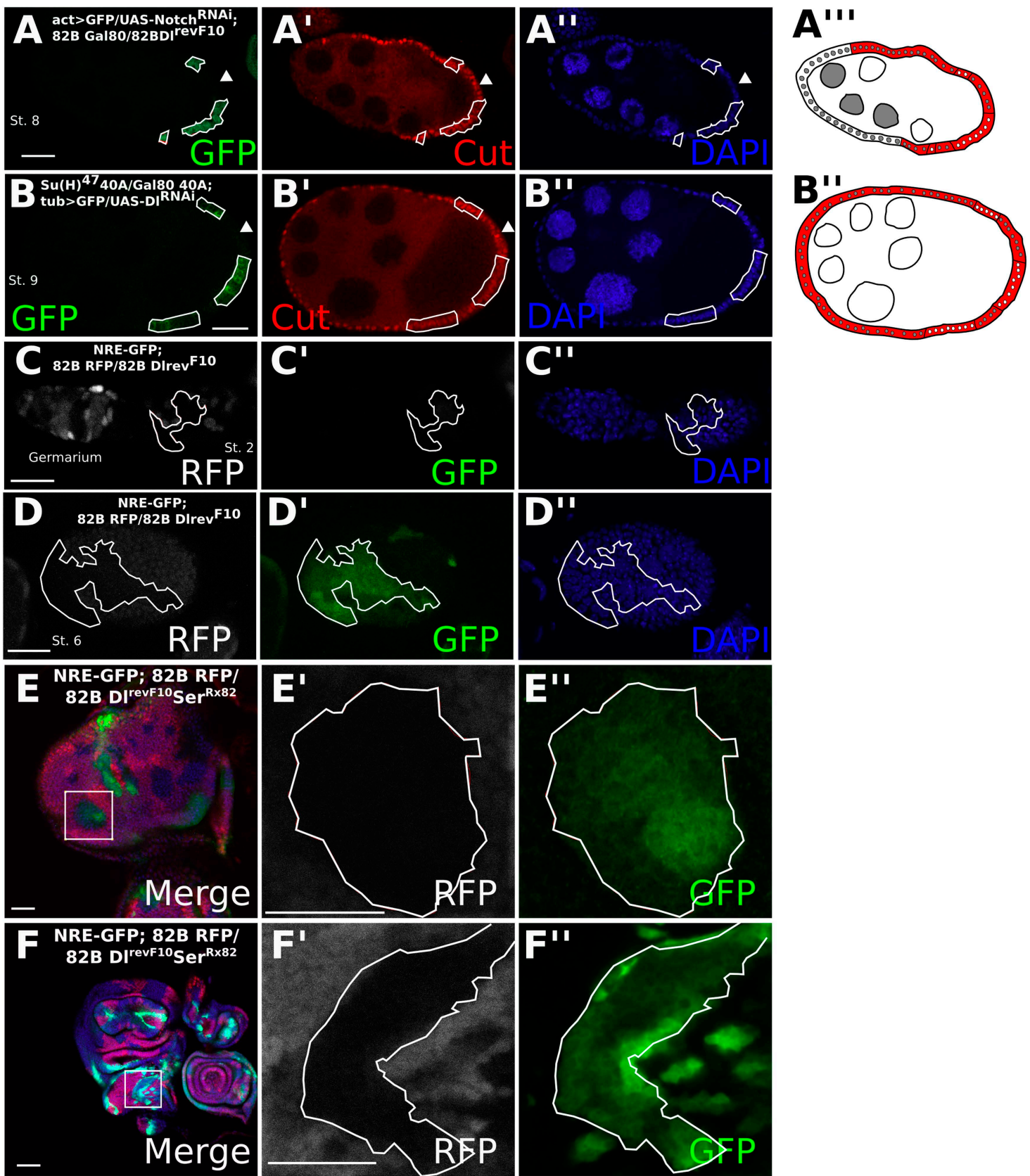

Figure 2. Cis-ligand represses ligand-independent Notch activity in the follicle cells and imaginal discs. D/revF10 mutant MARCM germline/follicle cell clones co-expressing Notch ${ }^{\text {RNAi }}$ show prolonged Cut expression (A). Su(H) $)^{47}$ MARCM mutant germline/follicle cell clones co-expressing D/RNAi show failure to enter the endocycle (B). Germline clones are shown by late Cut expression in wild-type follicle cells

(A, B, see arrowheads). See Figure 2-figure supplement 1A for control D/RNAi-induced germline follicle cell clones. Notch Responsive Elementgreen fluorescent protein (NRE-GFP) is upregulated beginning from stage 2 (C) and through later stages (D) in D/RevF10 germline and follicle cell clones. NRE-GFP is also upregulated cell-autonomously in $D /^{R e v F 10} S e r^{R \times 82}$ mutant clones in eye (E) and wing (F) imaginal discs. Scale bars represent $20 \mu \mathrm{m}$.

DOI: 10.7554/eLife.04415.006

The following figure supplement is available for figure 2:

Figure supplement 1. Control experiments relating to Figure 2. DOI: 10.7554/eLife.04415.007 
upregulation was spatially variable in the wing disc, having the highest prevalence in the notum region (25\% incidence), a low incidence in the dorsal pouch (8\%), whereas in the ventral pouch region it was never seen $(n=80)$ (Supplementary file 1), perhaps owing to the differential regulation of Notch degradation throughout the wing disc (Hori et al., 2011). As reported previously, most wing disc clones showed a higher NRE-GFP upregulation in the clone boundary where there is access to trans-ligand, indicating that the ligand-independent Notch activity observed occurs at a rather low level.

Drosophila S2 cells are reported to have no DI expression and a very low level of Ser expression, which had no effect on Notch signaling (Fehon et al., 1990; Graveley et al., 2011) (Figure 3-figure supplement 1), and have been used as a model to study ligand-independent Notch activity (Hori et al., 2011). Upon transfection with $p M T-N^{F L}$, a $\mathrm{CuSO}_{4}$-inducible full-length Notch construct, Notch activation was increased by a factor of 5.13 compared with the control cells, as indicated by a NRE-firefly luciferase reporter gene ( $<$ 0.0001) (Figure 3C). Notch activation in S2 cells is at least partially dependent on endosomal trafficking, as double-stranded (ds) RNA against early endosome component, Rab5, or multivesicular body sorting protein, hrs, reduced the levels of Notch activation (Figure 3A,B). This is consistent with the in vivo studies indicating that ligand-independent Notch activation relies heavily on receptor trafficking (Hori et al., 2012) (Rab5 $p=0.00623$, hrs $p=0.0159$ ), and our observation that Notch accumulates in DI-/DI- clones (Figure 3-figure supplement 2). A requirement for trafficking is consistent with the results of others who have demonstrated aberrant Notch activation in follicle cell mutants for trafficking components (Wilkin et al., 2004; Vaccari et al., 2008; Schneider et al., 2013), such as tsg101 mutant clones, which show early Notch activation in the follicle cells (Figure 3-figure supplement 3). Furthermore, co-transfecting pMT-N ${ }^{F L}$ with pMT-GAL4 and pUASt-Serdel3, a form of Ser that cannot activate Notch, but only cis-inhibit, (Fleming et al., 2013) almost entirely abolished the Notch activation detected when $N^{F L}$ was transfected alone $(p=0.0048)$ (Figure $3 C$ ). These results suggest that if Notch is expressed in a cell free of cis- and trans-ligands, DSL ligand-independent activity will occur and that cis-inhibition is extremely efficient in preventing this 'accidental' Notch activity as it travels through the endosomal pathway en route to degradation.

We next explored whether cis-inhibition can also block ligand-independent Notch activity induced in aberrant genetic backgrounds. The Notch target, Wingless $(\mathrm{Wg})$ is normally expressed along the dorsoventral boundary of the wing disc (Figure 4A). Lethal giant disc (lgd) homozygous mutant (lgd d7) larvae display overgrown imaginal discs and ubiquitous ligand-independent Notch activation in the wing pouch region, as shown by upregulation of Wg (Figure 4B). Notch activation in lgd mutant cells is caused by a defect in Notch trafficking and degradation, as the receptor is aberrantly transported to the limiting membrane of the lysosome which facilitates production of $\mathrm{N}^{\mathrm{ICD}}$ (Childress et al., 2006; Gallagher and Knoblich, 2006; Jaekel and Klein, 2006; Schneider et al., 2013). Using dpp-GAL4 to misexpress UAS-DI along the anterior-posterior axis of the wing disc in lgd ${ }^{d 7}$ homozygous larvae, Wg expression was considerably reduced along the dpp expression domain, indicating that cis-inhibition can block the ligand-independent Notch activity observed in this situation (Figure 4C). Overexpression of Deltex $(D x)$, an E3 ubiquitin ligase that stimulates Notch monoubiquitination and promotes its trafficking to the lysosomal limiting membrane, has also been shown to induce ligand-independent Notch activation specifically in the ventral wing pouch region (Matsuno et al., 2002; Hori et al., 2004; Wilkin et al., 2008; Schneider et al., 2013) (Figure 4D). We used patched (ptc)-GAL4 to drive expression of UAS-Dx with either UAS-DI or UAS-Serdel3, whose ectopic expression leads to a reduction of Wg staining along the dorsoventral boundary (Micchelli et al., 1997; Fleming et al., 2013) (controls in Figure 4figure supplement $1 A, E$ ). Co-expression of $D x$ and $D /$ led to a decrease in $W g$ expression in the ventral ptc domain as compared with expression of Dx alone (Figure 4E). When UAS-Dx and UAS$\mathrm{Ser}^{\text {del3 }}$ were co-expressed, there was a small but noticeable, albeit variable, decrease in Dx-induced Notch activation (Figure 4-figure supplement 1B-D). This incomplete reduction was probably due to the previously noted, slightly compromised, cis-inhibitory potential of UAS-Ser ${ }^{\text {del3 }}$ (Fleming et al., 2013) (Figure 4-figure supplement 1A). Taken together, these results provide evidence that cisligand has a negative effect on the raised levels of DSL-ligand independent Notch activation incurred in genetically abnormal cells.

To quantify this effect, we co-transfected $p M T-D x$ with $p M T-N^{F L}$, causing an increase by a factor of $4.21(p=0.0021)$ in the Notch activation compared with transfecting $p M T-N^{F L}$ alone (Figure 4F). Transfection of $p M T-N^{F L}$, $p M T-D x, p M T-G A L 4$, and $p U A S t-S e r^{d e / 3}$ significantly $(p=0.0194)$ reduced the level of Notch activation (Figure 4F). We next treated cells with dsRNA for either lgd or shrub (a component of the ESCRT-III complex). Lgd dsRNA induced an increase in Notch activation by a factor of 


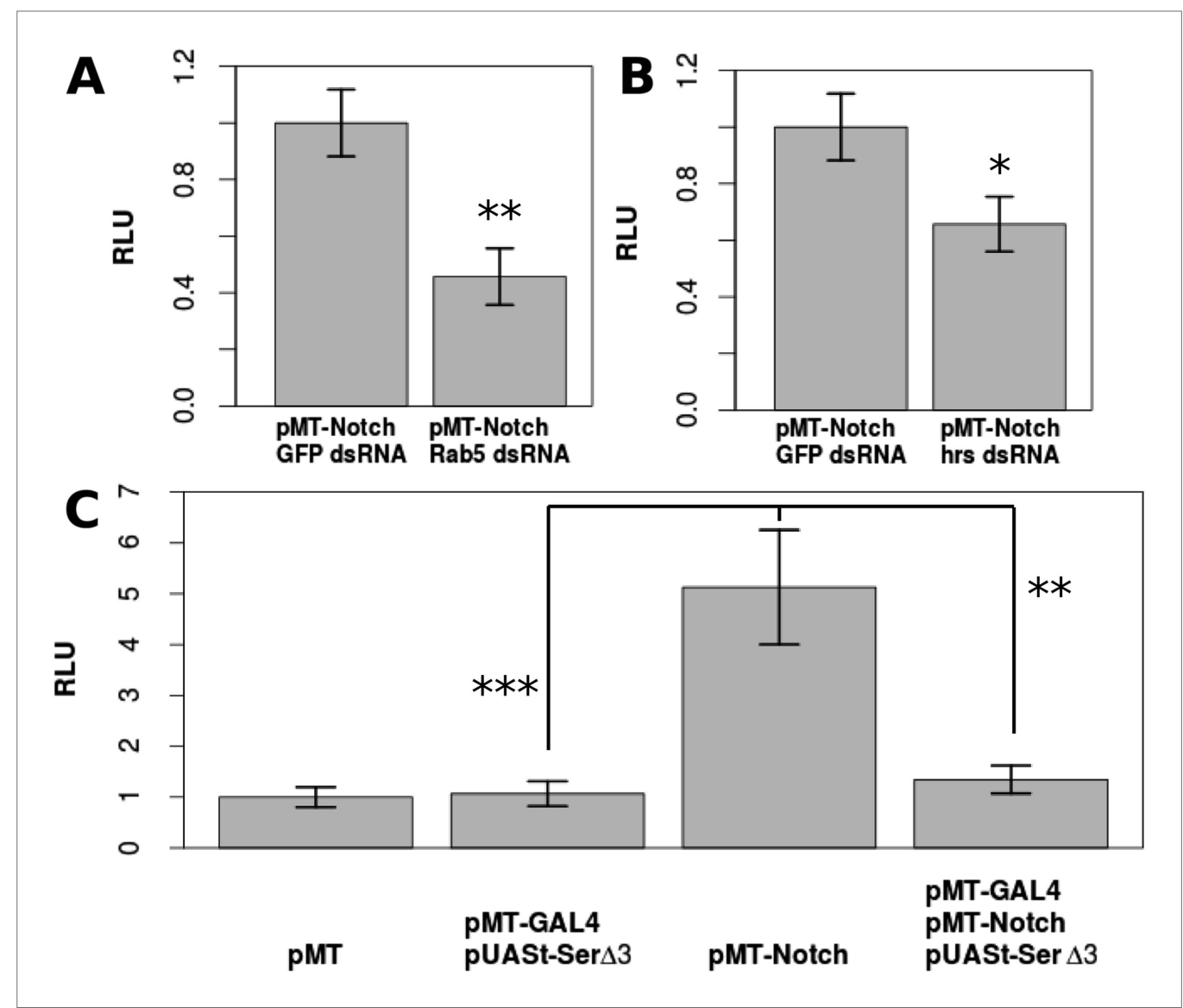

Figure 3. DSL-ligand-independent Notch activity in S2 cells is buffered by cis-ligand. Trafficking is important for Notch activation in S2 cells, as treatment with Rab5 dsRNA (A) or hrs dsRNA (B) significantly decreases the amount of Notch activated in S2 cells as shown by Notch-responsive luciferase activity (NRE-firefly) in relative light units (RLU). Transfecting only PMT-Notch ${ }^{F L}$ into S2 cells causes a 5.13-fold increase in Notch activation, which is almost entirely reduced (1.34-fold from the negative control) by co-transfection of pMT-GAL4 and pUASt-Serde/3 (C). Each experiment was carried out with two technical replicates and three biological replicates. Means of the technical replicates were used to carry out a paired t-test $(n=3)$ for each comparison. Error bars represent standard deviation (SD).

DOI: 10.7554/eLife.04415.008

The following figure supplements are available for figure 3:

Figure supplement 1. Addition of Ser dsRNA had no effect on the Notch activation in S2 cells in comparison with cells treated with control green fluorescent protein (GFP) dsRNA, indicating that the small amount of Ser expression is either not translated or does not significantly contribute to Notch activation upon transfection with pMT-NFL. DOI: 10.7554/eLife.04415.009

Figure supplement 2. Notch accumulates in DI-/DI- clones. DOI: 10.7554/eLife.04415.010

Figure supplement 3. Follicle cells mutant for ESCRT component tsg101 show early Notch activity in the follicle cells (Vaccari et al., 2008).

DOI: 10.7554/eLife.04415.011

1.73 compared with GFP dsRNA-treated cells $(p=0.00286$ ) (Figure 4G). Likewise, shrub dsRNA caused a 3.93-fold increase ( $p$ 0.0001) in Notch activation in S2 cells (Figure 4H) (Thompson et al., 2005). Expression of Serdel3 in both situations led to a significant decrease in the amount of Notch activated in comparison with Notch-expressing cells treated with control dsRNA (lgd $p=0.0093$, shrub $p=0.0257$ ) (Figure 4G,H).

To explore whether cis-acting ligands might block endogenous raised levels of ligand-independent Notch activation, in addition to the raised levels induced by genetic defects, we examined the effect of increased ligand expression in crystal cells in the larval lymph gland, which have recently been shown 

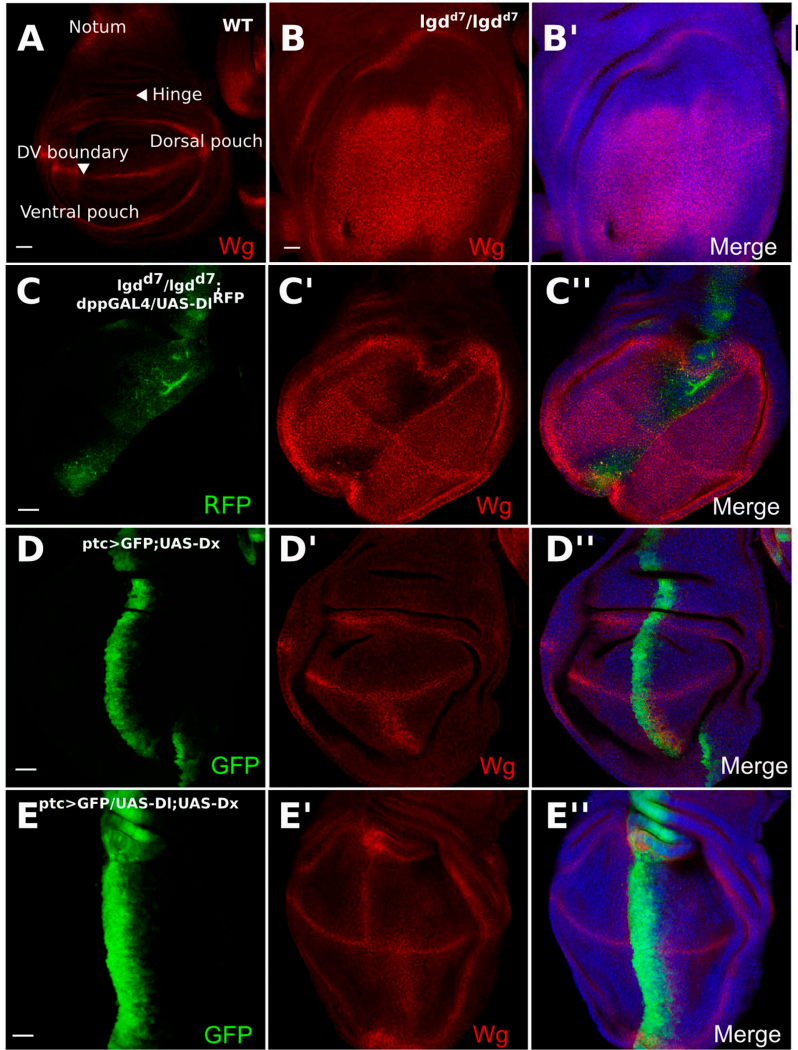
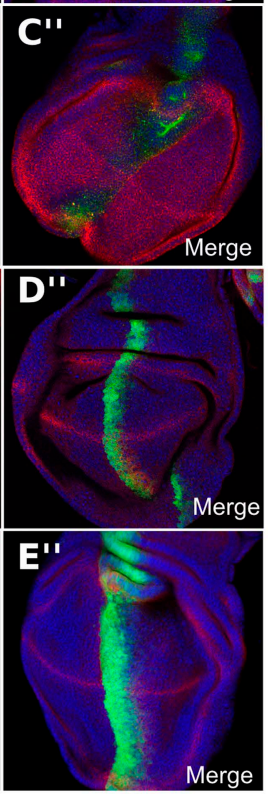
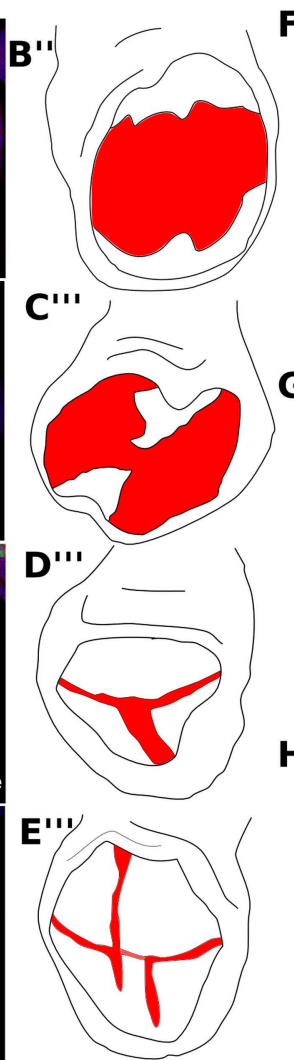

$\mathbf{F}$

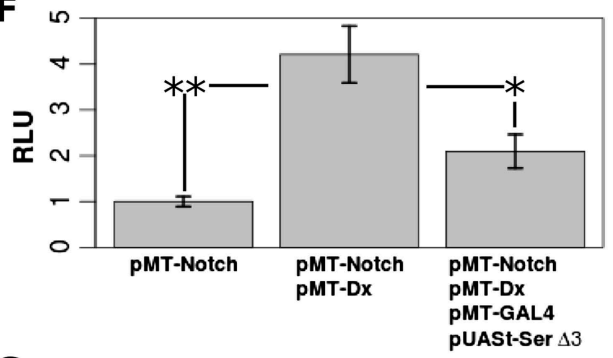

G
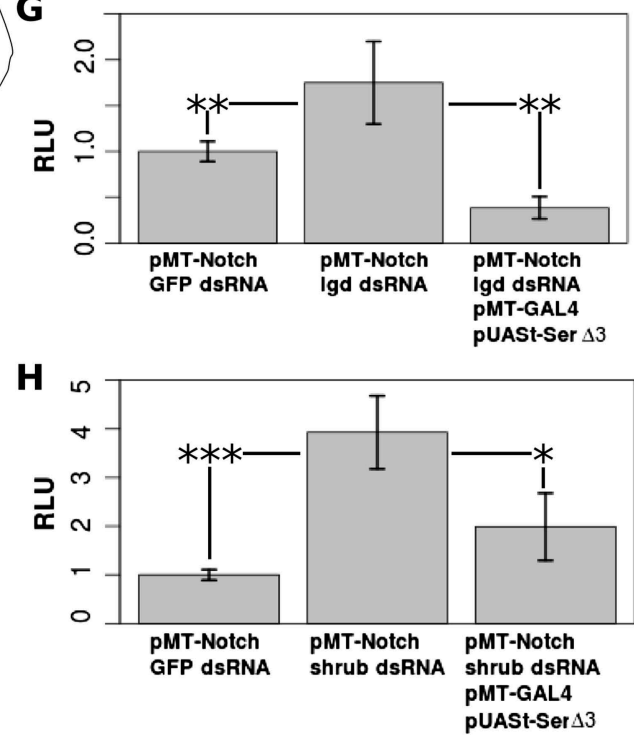

Figure 4. Notch ligand buffers against genetically induced DSL-independent activation. Wing discs were stained with Wg antibody and illustrations are colored red where Wg is expressed (A-E). A wing disc with regions of interest is labeled and WT Wg staining shown (A). Igdd7/lgdd7 wing discs show ubiquitous $W g$ expression in the wing pouch as a result of DSL-ligand-independent Notch activity (B). Misexpression of UAS-D/ in lgdd7/lgd ${ }^{d 7}$ discs causes a reduction in Wg staining along the anteroposterior boundary of the pouch (C). ptcGAL4 drives UAS-Dx causing ectopic Notch activity in the ventral wing pouch (D). Co-expression of Dx with D/ reduces Wg staining in the ptc domain $(\mathbf{E})$, although, as in $/ g d^{d 7} / \lg d^{d 7}$ discs, the reduction is not complete towards the dorsoventral boundary. Cis-ligand also decreases Notch activation caused by genetic defects in S2 cells (F-H). Co-transfection with $P M T-N^{F L}$ and PMT-Dx caused a significant increase in Notch luciferase reporter expression, and adding Serdel3 significantly reduced this Dx-induced activation (F). Cells treated with Igd dsRNA (G) or ESCRT-III component, shrub, dsRNA (H) also caused significant increases in Notch reporter activity, either of which could be blocked by addition of Serdel3. For each of the S2 cell experiments, means were taken for technical duplicates and used for a paired t-test for three biological replicates. Error bars represent SD. Scale bars represent $20 \mu \mathrm{m}$.

DOI: 10.7554/eLife.04415.012

The following figure supplements are available for figure 4:

Figure supplement 1. Co-expression of UAS-Dx and UAS-Serdels has a variable effect on DSL-independent Notch activation. DOI: 10.7554/eLife.04415.013

Figure supplement 2. Endogenous DSL-independent Notch activity in crystal cells is reduced by cis-inhibition.

DOI: 10.7554/eLife.04415.014

Figure supplement 3. Reduced Notch reporter activity in crystal cells was not caused by indirect effects on early ligand-dependent Notch signaling in prohaemocytes.

DOI: 10.7554/eLife.04415.015

to have ligand-independent Notch activation (Mukherjee et al., 2011). Notch activity in crystal cells promotes cell survival, and decreased Notch activity leads to a 'bursting' phenotype (Mukherjee et al., 2011) (Figure 4-figure supplement 2B,E). Evidence for this bursting phenotype is provided by the disorganization of membrane-associated GFP (Mukherjee et alı, 2011). Using Lozenge (Lz)-GAL4, a crystal cell lineage-specific driver (Terriente-Felix et al., 2013) to misexpress UAS-Notch ${ }^{\text {RNAi }}$ or UASSerde/3 led to a significantly higher proportion of cells showed the 'bursting' phenotype than wild-type crystal cells (Notch ${ }^{R N A i} p=0.0434$, Ser $^{\text {del3 }} p=0.0286$ ) (Figure 4-figure supplement 2A,B,E). Furthermore, 
overexpression of UAS-Ser ${ }^{W T}$ led to a significant decrease of the Notch reporter $E(s p l): m \beta-C D$ 2expression in mature crystal cells (Figure 4-figure supplement 2C,D,F). Reduced Notch reporter activity was not caused by indirect effects on early ligand-dependent Notch signaling in prohaemocytes, as Hnt, a Notch target in differentiating crystal cells, (Terriente-Felix et al., 2013) was unaffected by ligand misexpression (Figure 4-figure supplement 3A,B). These observations indicate that increased ligand expression in crystal cells decreases cell survival by blocking Notch ligand-independent activation, and therefore the buffering role of cis-expressed ligand can be extended to endogenous cases of DSLindependent Notch activity.

In this study, we show that cells devoid of DSL ligands activate Notch sufficiently to stimulate reporter activity, and in the ovarian follicle cells the level of activation is above the threshold required to mediate normal Notch-induced downstream developmental events. During development, this type of noncanonical Notch activity is normally prevented by cis-expressed DSL ligands in numerous tissues. Cis-inhibition can also attenuate DSL-ligand independent Notch activity both in endogenous and genetically induced situations. Mechanistically, this could be explained if DSL ligands sequestered Notch at the membrane, made Notch more sensitive to degradation, or increased the stability of the heterodimer as it travels through the endosomal pathway. As we and others (Fiuza et al., 2010) have shown that increasing or decreasing ligand has variable effects on receptor distribution among tissues, and given that we observe a consistent effect among tissues on Notch activation upon cis-ligand removal, we prefer the stability hypothesis. Fiuza et al. (2010) show that ligand affects Notch stability during Notch activation by EDTA, giving support to the stability hypothesis as the most parsimonious explanation (Fiuza et al., 2010). It is suggested that retaining a pool of translated Notch receptor keeps the pathway in a condition capable of almost instant activation (Sprinzak et al., 2010). Therefore, we propose that a role of cis-ligands might be to keep the Notch pathway in a state of readiness by buffering against unintentional stochastic Notch activity resulting from normal processing through the endosomes. Endogenously, this may aid the ability of a cell to mediate future Notch-dependent developmental events that have strict temporal regulation.

\section{Materials and methods}

\section{Drosophila stocks and generation of clones}

The following fly stocks were used for Drosophila crosses. hs-flp ${ }^{122}$;FRT82B RFP (Poulton et al., 2011), FRT82B DIRevF10 (Haenlin et al., 1990), FRT82B D|RevF10Ser ${ }^{R \times 82}$ (BDSC \#6300), hs-FLP122; act-GAL4 UASGFP;FRT82B Gal80, UAS-Notch ${ }^{\text {RNAi }}$ (VDRC \#1112-no expression in germline cells), UAS-Delta ${ }^{\text {RNAi }}$ (BDSC \#34322-able to express in germline cells); hsFLP GFPstau; act > y+ > GAL4, UAS-GFP, hs-flp ${ }^{122 ;}$; Gal80 FRT40A; tubGAL4 UASGFP, Su(H)47FRT40A (Morel and Schweisguth, 2000), NRE-EGFP (BDSC \#30727; Stempfle et al., 2010), ubx-FLP;;FRT82B RFP, patched-GAL4 UAS-GFP (Hinz et al., 1994), UAS-DIMyc (a gift from Marc Muskavitch), tsg101111019 from Kyoto stock center, UAS-Ser'WT (BDSC \#5815), UAS-Serdel3-tom (a gift from Robert J Fleming) (Graveley et al., 2011), UAS-Deltex (a gift from Martin Baron), lgd d740A (BDSC \#25087), dppGAL4 (BDSC \#7007), Iz-GAL4 UAS-GFP (BDSC \#6314). To create FRT82B, DlRevF10 germline/ follicle cell clones by the FLP/FRT or MARCM methods (Golic and Lindquist, 1989; Lee and Luo, 2001) (e.g., Figures 1B,D-F, 2A,C-D, Figure 1-figure supplement 2A-B), crossed flies were subjected to a $2 \mathrm{hr}$ heat shock at $37^{\circ} \mathrm{C}$ for two consecutive days while in the mid-pupal to late-pupal stages. Flies were sorted three days after eclosion, and then kept for an extra three days at $25^{\circ}$ before an additional 1-hr heat shock and incubation at $29^{\circ} \mathrm{C}$ with yeast paste for two more days before dissection. FLP-out-induced D/RNAi germline/follicle cell clones (e.g., Figure 2B, Figure 2-figure supplement 1A,B) were produced by two consecutive 50 -min heat shocks, followed by incubation at $25^{\circ} \mathrm{C}$ for a week and then transfer to yeasted vials in the $29^{\circ} \mathrm{C}$ incubator for dissection two days later. Evidence for MARCM and FLP-out-induced germline clones was provided by small nuclei and late Cut expression, as the UASt-GFP transgene does not reliably express in the germline. Follicle cell clones alone were produced by two 50-min heat shocks, followed by two days' incubation at $29^{\circ} \mathrm{C}$ (e.g., Figure $1 \mathrm{C}$ and Figure 2-figure supplement 1C-D). Imaginal disc FLPFRT-induced mutant clones were produced either by a ubx-FLP or a 1-hr heat shock with hs-FLP'22 two days after egg laying. All other crosses were kept at $25^{\circ} \mathrm{C}$ unless otherwise noted. In lymph gland studies, Grubbs' test was used to identify significant $(p<0.05)$ outliers, which were omitted from further analyses.

\section{Immunostaining}

Ovaries, imaginal discs, or lymph glands were dissected in phosphate-buffered saline (PBS), fixed in 10\% formaldehyde, washed three times in PBS + Triton-X (PBT), and then blocked for at least $1 \mathrm{hr}$ in 
PBT with goat serum. Tissues were then either stained overnight with mouse anti-Cut (DSHB 2B10, 1:30), mouse anti-Hindsight (DSHB 1G9, 1:15), mouse anti-NICD (DSHB C179C6, 1:15), mouse anti-NECD (DSHB C4582H, 1:15), mouse anti-Wingless (DSHB 4D4, 1:20), mouse anti-DI (DSHB C594.9B, 1:15), rabbit anti- $\beta$ Gal (MP Biomedical, Santa Ana, CA. SKU \#08559761), or rabbit anti-GFP (abcam, Cambridge, UK. ab290-NRE-GFP was co-stained with this antibody to increase reporter sensitivity) primary antibodies. Tissues were mounted on slides after PBT washes and secondary antibody incubation. 4',6-Diamidino-2-phenylindole (DAPI) was used to stain nuclei. Samples were then analyzed with a Zeiss 510 or Leica SP2 confocal microscope and after analysis with the Image J software. Nuclear volume quantification was done with the Volumest plug-in for ImageJ.

\section{S2 cell transfection and RNA interference}

S2 cells were grown under standard conditions and passaged once every three days in serum-free Gibco media (Invitrogen, Waltham, MA) supplemented with antibiotics. In preparation for transfection $10^{6}$ cells per milliliter were seeded into either 24-well plates or 96-well plates for experiments with or without dsRNA treatment, respectively. Transfections were carried out with Qiagen Effectene (Qiagen, Netherlands) transfection reagent according to the manufacturer's instruction. Plasmids used for transfection were pMT-Notch ${ }^{\mathrm{FL}}$ (a gift from Renjie Jiao), pMT-GAL4 (DGRC \#1042), pUASt-Serdel3 (a gift from Robert J Fleming), pMT-Deltex (a gift from Spyros Artavanis-Tsakonas), NRE-firefly luciferase (a gift from Sarah Bray), or Renilla luciferase (a gift from Sarah Bray). Aliquots (75 ng for 24-well plates or $50 \mathrm{ng}$ for 96-well plates) of each non-luciferase plasmid were added and, where applicable, $10 \mathrm{ng}$ of each luciferase plasmid. DNA concentration between transfections was kept constant with an empty vector. For experiments without dsRNA treatment, $\mathrm{CuSO}_{4}$ was added to a concentration of $500 \mu \mathrm{M} 24 \mathrm{hr}$ after transfection, and cells were assayed $24 \mathrm{hr}$ later. dsRNA was transcribed in vitro using the RiboMAX large-scale RNA production system-T7 kit (Promega, Madison, WI). The following primers were used to amplify genomic DNA taken from a single male fly from the NRE-GFP stock:

\section{Rab5}

Forward: GAATTAATACGACTCACTATAGGGCAGGGGACGAATTTCATTTG

Reverse: GAATTAATACGACTCACTATAGGGAAAACCCTGCGCTTTCTTCT

Hrs

Forward: GAATTAATACGACTCACTATAGGGAATCGCCAACAATCAAGTCC

Reverse: GAATTAATACGACTCACTATAGGGCGTGCAGCACTACTTTCCAA

Lgd

Forward: GAATTAATACGACTCACTATAGGGAGATGCCTCTGAGGAACCCGTCCAG

Reverse: GAATTAATACGACTCACTATAGGGAGAGTGTGGGTTCTGGGGCAGCAGT

Shrub

Forward: GAATTAATACGACTCACTATAGGGACTTTTATGCAGGGACGTGG

Reverse: GAATTAATACGACTCACTATAGGGTCCCTCGCTTCGAACTAAAA

Serrate

Forward: GAATTAATACGACTCACTATAGGGTCTCACCAACCAACCAATCA

Reverse: GAATTAATACGACTCACTATAGGGCACAATATAGAGCGCGACGA

GFP

Forward: GAATTAATACGACTCACTATAGGGAGCTGGACGGCGACGTAAAC

Reverse: GAATTAATACGACTCACTATAGGGATGGGGGTGTTCTGCTGGTAG

Cells were treated with dsRNA at a concentration of $50 \mathrm{nM}$, and then transfected shortly after. $\mathrm{CuSO}_{4}$ was added to a concentration of $500 \mu \mathrm{M}$ later that day. Cells were incubatedfor five days, with an additional treatment of dsRNA on the fourth day.

\section{Luciferase assay}

Cells were transfected with plasmids of interest together with an NRE-driving firefly luciferase expression and a constitutively activated Renilla luciferase to control for transfection efficiency. Luciferase measures were inspected with the Dual-Luciferase Assay Kit (Promega) in 96-well luminometer plates. Each transfection was performed in duplicate and repeated several times. Student's $t$ test was used to test for statistical significance. 


\section{Acknowledgements}

Dongyu Jia discovered the phenotype of Hindsight, and Cut expression in DI-/DI- (DI germline/ follicle cell) clones, and developed the project as DSL ligand-independent mitotic cycle/endocycle switch initially. We thank Marc Muskavitch, Martin Baron, Robert Fleming, Renjie Jiao, Spyros ArtavanisTsakonas, Sarah Bray, the Bloomington Drosophila Stock Center, the Vienna Drosophila RNAi Center, the TRiP at Harvard Medical School, and the Developmental Studies Hybridoma Bank for providing us with stocks and reagents. We also thank Yi-Chun Huang for technical assistance, and Gary Struhl, John Poulton, Pang-Kuo Lo, Gengqiang Xie, Jen Kennedy, Steven Lenhert, and Gabriel Calvin for helpful comments and suggestions while preparing the manuscript. W-MD is supported by $\mathrm{NIH}$ grants R01GM072562 and NSF IOS-1052333.

\section{Additional information}

Funding

\begin{tabular}{lll} 
Funder & Grant reference number & Author \\
\hline National Science Foundation & IOS-1052333 & Wu-Min Deng \\
\hline National Institutes of Health & R01GM072562 & Wu-Min Deng
\end{tabular}

The funders had no role in study design, data collection, and interpretation, or the decision to submit the work for publication.

Author contributions

WHP, Conception and design, Acquisition of data, Analysis and interpretation of data, Drafting or revising the article; DJ, Conception and design, Acquisition of data; W-MD, Conception and design, Analysis and interpretation of data, Drafting or revising the article, Contributed unpublished essential data or reagents

\section{Additional files}

Supplementary file

- Supplementary file 1. Supplementary clonal data file. Excel worksheet containing clonal data from the egg chamber and the wing disc.

DOI: 10.7554/eLife.04415.016

\section{References}

Childress JL, Acar M, Tao C, Halder G. 2006. Lethal giant discs, a novel c2-domain protein, restricts notch activation during endocytosis. Current Biology 16:2228-2233. doi: 10.1016/j.cub.2006.09.031.

de Celis JF. 2013. Understanding the determinants of notch interactions with its ligands. Science Signaling 6:pe19. doi: 10.1126/scisignal.2004079.

Del Álamo D, Rouault H, Schweisguth F. 2011. Mechanism and significance of cis-inhibition in notch signalling. Current Biology 21:R40-R47. doi: 10.1016/j.cub.2010.10.034.

Deng WM, Althauser C, Ruohola-Baker H. 2001. Notch-Delta signaling induces a transition from mitotic cell cycle to endocycle in Drosophila follicle cells. Development 128:4737-4746.

Fehon RG, Kooh PJ, Rebay I, Regan CL, Xu T, Muskavitch MA, Artavanis-Tsakonas S. 1990. Molecular interactions between the protein products of the neurogenic loci Notch and Delta, two EGF-homologous genes in Drosophila. Cell 61:523-534. doi: 10.1016/0092-8674(90)90534-L.

Fiuza UM, Klein T, Martinez Arias A, Hayward P. 2010. Mechanisms of ligand-mediated inhibition in Notch signaling activity in Drosophila. Developmental Dynamics 239:798-805.

Fleming RJ, Hori K, Sen A, Filloramo GV, Langer JM, Obar RA, Artavanis-Tsakonas S, Maharaj-Best AC. 2013. An extracellular region of Serrate is essential for ligand-induced cis-inhibition of Notch signaling. Development 140:2039-2049. doi: 10.1242/dev.087916.

Gallagher CM, Knoblich JA. 2006. The conserved c2 domain protein lethal (2) giant discs regulates protein trafficking in Drosophila. Developmental Cell 11:641-653.

Golic KG, Lindquist S. 1989. The FLP recombinase of yeast catalyzes site-specific recombination in the Drosophila genome. Cell 59:499-509. doi: 10.1016/0092-8674(89)90033-0.

Graveley BR, Brooks AN, Carlson JW, Duff MO, Landolin JM, Yang L, Artieri CG, van Baren MJ, Boley N, Booth BW Brown JB, Cherbas L, Davis CA, Dobin A, Li R, Lin W, Malone JH, Mattiuzzo NR, Miller D, Sturgill D, Tuch BB, 
Zaleski C, Zhang D, Blanchette M, Dudoit S, Eads B, Green RE, Hammonds A, Jiang L, Kapranov P, Langton L, Perrimon N, Sandler JE, Wan KH, Willingham A, Zhang Y, Zou Y, Andrews J, Bickel PJ, Brenner SE, Brent MR, Cherbas P, Gingeras TR, Hoskins RA, Kaufman TC, Oliver B, Celniker SE. 2011. The developmental transcriptome of Drosophila melanogaster. Nature 471:473-479. doi: 10.1038/nature09715.

Haenlin M, Kramatschek B, Campos-Ortega JA. 1990. The pattern of transcription of the neurogenic gene Delta of Drosophila melanogaster. Development 110:905-914.

Hinz U, Giebel B, Campos-Ortega J. 1994. The basic-helix-loop-helix domain of Drosophila lethal of scute protein is sufficient for proneural function and activates neurogenic genes. Cell 76:77-87. doi: 10.1016/0092-8674(94)90174-0.

Hori K, Fostier M, Ito M, Fuwa TJ, Go MJ, Okano H, Baron M, Matsuno K. 2004. Drosophila deltex mediates suppressor of Hairless-independent and late-endosomal activation of Notch signaling. Development 131:5527-5537. doi: 10.1242/dev.01448.

Hori K, Sen A, Kirchhausen T, Artavanis-Tsakonas S. 2011. Synergy between the ESCRT-III complex and Deltex defines a ligand-independent Notch signal. The Journal of Cell Biology 195:1005-1015. doi: 10.1083/jcb.201104146.

Hori K, Sen A, Kirchhausen T, Artavanis-Tsakonas S. 2012. Regulation of ligand-independent Notch signal through intracellular trafficking. Communicative \& Integrative Biology 5:374-376. doi: 10.4161/cib.19995.

Jaekel R, Klein T. 2006. The Drosophila notch inhibitor and tumor suppressor gene lethal (2) giant discs encodes a conserved regulator of endosomal trafficking. Developmental Cell 11:655-669. doi: 10.1016/j.devcel.2006.09.019.

Lee T, Luo L. 2001. Mosaic analysis with a repressible cell marker (MARCM) for Drosophila neural development. Trends in Neurosciences 24:251-254. doi: 10.1016/S0166-2236(00)01791-4.

López-Schier H, St Johnston D. 2001. Delta signaling from the germ line controls the proliferation and differentiation of the somatic follicle cells during Drosophila oogenesis. Genes \& Development 15:1393-1405. doi: 10.1101/gad.200901.

Matsuno K, Ito M, Hori K, Miyashita F, Suzuki S, Kishi N, Artavanis-Tsakonas S, Okano H. 2002. Involvement of a proline-rich motif and RING-H2 finger of Deltex in the regulation of Notch signaling. Development 129:1049-1059.

Micchelli CA, Rulifson EJ, Blair SS. 1997. The function and regulation of cut expression on the wing margin of Drosophila: notch, Wingless and a dominant negative role for Delta and Serrate. Development 124:1485-1495.

Morel V, Schweisguth F. 2000. Repression by suppressor of hairless and activation by Notch are required to define a single row of single-minded expressing cells in the Drosophila embryo. Genes \& Development 14:377-388.

Mukherjee T, Kim WS, Mandal L, Banerjee U. 2011. Interaction between Notch and Hif- $\alpha$ in development and survival of Drosophila blood cells. Science 332:1210-1213. doi: 10.1126/science.1199643.

Poulton JS, Huang YC, Smith L, Sun J, Leake N, Schleede J, Stevens LM, Deng WM. 2011. The microRNA pathway regulates the temporal pattern of Notch signaling in Drosophila follicle cells. Development 138:1737-1745. doi: 10.1242/dev.059352.

Schneider M, Troost T, Grawe F, Martinez-Arias A, Klein T. 2013. Activation of Notch in lgd mutant cells requires the fusion of late endosomes with the lysosome. Journal of Cell Science 126:645-656. doi: 10.1242/jcs.116590.

Sprinzak D, Lakhanpal A, Lebon L, Santat LA, Fontes ME, Anderson GA, Garcia-Ojalvo J, Elowitz MB. 2010. Cis-interactions between Notch and Delta generate mutually exclusive signalling states. Nature 465:86-90. doi: 10.1038/nature08959.

Stempfle D, Kanwar R, Loewer A, Fortini ME, Merdes G. 2010. In vivo reconstitution of gamma-secretase in Drosophila results in substrate specificity. Molecular and Cellular Biology 30:3165-3175. doi: 10.1128/ MCB.00030-10.

Sun J, Deng WM. 2005. Notch-dependent downregulation of the homeodomain gene cut is required for the mitotic cycle/endocycle switch and cell differentiation in Drosophila follicle cells. Development 132:4299-4308. doi: 10.1242/dev.02015.

Sun J, Deng WM. 2007. Hindsight mediates the role of notch in suppressing hedgehog signaling and cell proliferation. Developmental Cell 12:431-442. doi: 10.1016/j.devcel.2007.02.003.

Terriente-Felix A, Li J, Collins S, Mulligan A, Reekie I, Bernard F, Krejci A, Bray S. 2013. Notch cooperates with Lozenge/Runx to lock haemocytes into a differentiation programme. Development 140:926-937. doi: 10.1242/ dev.086785.

Thompson BJ, Mathieu J, Sung HH, Loeser E, Rørth P, Cohen SM. 2005. Tumor suppressor properties of the ESCRT-II complex component Vps25 in Drosophila. Developmental Cell 9:711-720. doi: 10.1016/j. devcel.2005.09.020.

Vaccari T, Lu H, Kanwar R, Fortini ME, Bilder D. 2008. Endosomal entry regulates Notch receptor activation in Drosophila melanogaster. The Journal of Cell Biology 180:755-762. doi: 10.1083/jcb.200708127.

Wilkin MB, Carbery AM, Fostier M, Aslam H, Mazaleyrat SL, Higgs J, Myat A, Evans DA, Cornell M, Baron M. 2004. Regulation of notch endosomal sorting and signaling by Drosophila Nedd4 family proteins. Current Biology 14:2237-2244. doi: 10.1016/j.cub.2004.11.030.

Wilkin M, Tongngok P, Gensch N, Clemence S, Motoki M, Yamada K, Hori K, Taniguchi-Kanai M, Franklin E, Matsuno K, Baron M. 2008. Drosophila HOPS and AP-3 complex genes are required for a Deltex-regulated activation of Notch in the endosomal trafficking pathway. Developmental Cell 15:762-772. doi: 10.1016/j. devcel.2008.09.002. 